\title{
A RBFNN-Based Adaptive Disturbance Compensation Approach Applied to Magnetic Suspension Inertially Stabilized Platform
}

\author{
Quanqi Mu, ${ }^{1,2}$ Gang Liu, ${ }^{1,2}$ and Xusheng Lei ${ }^{1,2}$ \\ ${ }^{1}$ Science and Technology on Inertial Laboratory, Beihang University, Beijing 100191, China \\ ${ }^{2}$ School of Instrument Science and Opto-Eletronics Engineering, Beihang University, Beijing 100191, China \\ Correspondence should be addressed to Quanqi Mu; muquanqi369@163.com
}

Received 5 March 2014; Revised 11 June 2014; Accepted 11 June 2014; Published 3 August 2014

Academic Editor: Yi Chen

Copyright (c) 2014 Quanqi Mu et al. This is an open access article distributed under the Creative Commons Attribution License, which permits unrestricted use, distribution, and reproduction in any medium, provided the original work is properly cited.

\begin{abstract}
Compared with traditional mechanical inertially stabilized platform (ISP), magnetic suspension ISP (MSISP) can absorb high frequency vibrations via a magnetic suspension bearing system with five degrees of freedom between azimuth and pitch gimbals. However, force acting between rotor and stator will introduce coupling torque to roll and pitch gimbals. Since the disturbance of magnetic bearings has strong nonlinearity, classic state feedback control algorithm cannot bring higher precision control for MSISP. In order to enhance the control accuracy for MSISP, a disturbance compensator based on radial basis function neural network (RBFNN) is developed to compensate for the disturbance. Using the Lyapunov theorem, the weighting matrix of RBFNN can be updated online. Therefore, the RBFNN can be constructed without priori training. At last, simulations and experiment results validate that the compensation method proposed in this paper can improve ISP accuracy significantly.
\end{abstract}

\section{Introduction}

As the middle mechanism between aircraft and remote sensing payload, inertially stabilized platform (ISP) supports the payload, isolates aircraft movements (angular motion and linear motion), and holds the line-of-sight (LOS) of the payload vertically steady relative to earth [1-5]. It plays an important role in the remote sensing image quality. Therefore, GSM3000, SSM-270, SSM-350 [6, 7], PAV30, PAV80, PAV100 $[8,9]$, and T-AS mount $[10]$ are developed in recent years.

However, most of the ISPs are suspended by mechanical bearings. Mechanical ISP absorbs high frequency vibrations passively via four dampers, but there is still a small proportion of residual disturbance that is transmitted to the payload through mechanical bearings. Therefore, the imaging quality is deteriorated. Magnetic suspension inertially stabilized platform (MSISP), which has the characteristic of frictionless and active vibration suppression capacity, can further enhance imaging quality. However, force acting between rotor and stator will introduce coupling torque to roll and pitch gimbals, increasing difficulty in gimbal control of ISP.
Currently, ISP control methods can be divided into three categories: (1) PID-based or intelligent PID control [11-13]. They are widely applied in practical engineering, but generally the control accuracy and antidisturbance capacity are not satisfactory. (2) Control methods based on disturbance compensation, attenuation, and rejection; feed-forward control, disturbance observer based control, and $H_{\infty}$-based control, the typical literatures are listed as follows. Literature $[3,4]$ presented an acceleration feed-forward method to compensate for the disturbing torque caused by the linear acceleration of the carrier. But the signal of the accelerometer may be easily distorted by centripetal acceleration. In literature [14], a reduced-order disturbance observer (DOB) for line-of-sight control system was proposed. It presented a straightforward generic design approach and considered system parameter sensitivity and engineering implementation. Although the simulations validated system performance and robustness, the noise coupling would be potentially introduced by the observer. Literature [15] introduced an $H_{\infty}$ /predictive output control method for a three-axis gyrostabilized platform, and in the severe disturbance environment the proposed controller worked better than nonlinear proportion integration 
differentiation (NLPID) controller. Unfortunately, complex design procedure limited its practical applications. Compared with the PID-based control, the control methods based on disturbance compensation, attenuation, and rejection could enhance the accuracy to some extent, but they are dependent on system model. (3) Advanced intelligent control: fuzzy control [16, 17] sliding mode control [18]. In recent years, neural-network-based control has been widely used in induction motor control $[19,20]$ and multiaxis motion control [21]. It can get much more attractive performance and robustness than conventional control method. However, it needs a lot of time and data to train the weighting of the neural network.

In order to enhance the accuracy and the environmental adaptability, a new RBFNN-based adaptive control approach for MSISP is proposed. It can estimate and eliminate disturbance effectively to improve control performance. At last, simulations and experiment results prove that the RBFNNbased adaptive control approach proposed can lead to precise control performance for the MSISP system.

\section{Working Principle of the MSISP}

Figure 1 shows the structure of three-axis MSISP. There are three gimbals: roll gimbal (outer gimbal), pitch gimbal (middle gimbal), and azimuth gimbal (inner gimbal). Both the roll gimbal and the pitch gimbal use indirect drive method (motor plus a pair of gears), and bearings of the two gimbals are mechanical bearings. As for the azimuth gimbal, it uses direct drive with a brushless DC permanentmagnetic synchronous motor, and its bearing is a maglev bearing. Gimbal servo controller generates control signals to drive the motors according to position and attitude provided by POS (position and orientation system), as well as the angular rate measured by rate gyro $G_{x y}$ and $G_{z}$. Torque produced by motors compensates for outer disturbances and rotates the gimbals, thus making payload LOS tracking command position and attitude. Maglev bearing controller generates coil current control signals to adjust the distance between rotor and stator for actively absorb the residual high frequency vibrations.

Because the bandwidth of the maglev bearing control system is far beyond that of the gimbal control system, besides, based on PID control method, the angular error between the maglev bearing stator and rotor can be controlled within $0.003^{\circ}$ that can be ignored, the gimbal control system can be considered to be independent of the maglev bearing system, and the force between the maglev bearing stator and rotor is considered as disturbance to the gimbal system.

According to the typical photo flight situation, the dynamic rotation range of the base plate is within $\pm 5^{\circ}$. Furthermore, the aircraft angular rate is less than $10^{\circ} / \mathrm{s}$ under typical aircraft photo flight frequency spectrum $(\leq 0.5 \mathrm{~Hz})$ $[6,8]$, so the coupling torque among gimbals and the comprehensive influence from the base plate to each gimbal can be ignored $[4,22]$. Therefore, the controller for gimbal can be designed separately.

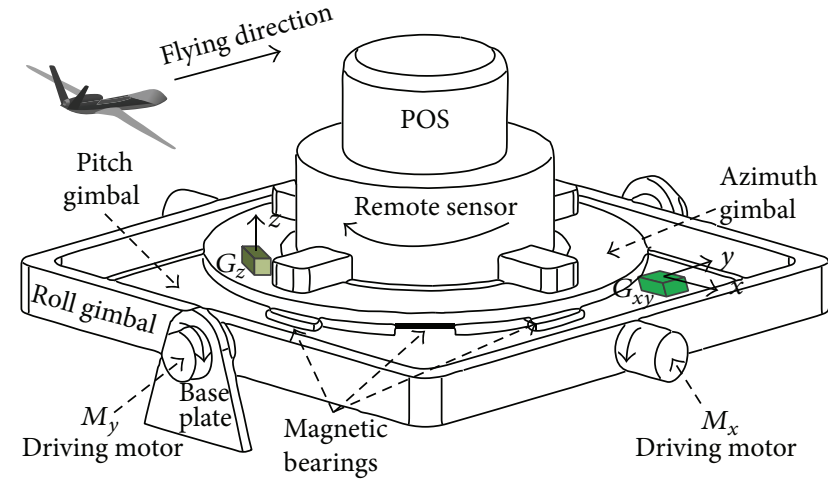

FIGURE 1: MSISP configuration diagram.

As for a single ISP gear-gimbal assembly driven by a DC torque motor with a pair of gears, its model can be described as follows:

$$
\begin{aligned}
& \omega_{L}(s) \\
& =\left(N K_{t} U(s)+\left(N T_{d m}+T_{d L}\right)\left(L_{m} s+R_{m}\right)\right. \\
& \left.+\left[N(N-1) J_{m} s+N^{2} K_{t} K_{e}\right] \omega_{b}(s)\right) \\
& \times\left(s\left(L_{m} s+R_{m}\right)\left(J_{L}+N^{2} J_{m}\right)+N^{2} K_{t} K_{e}\right)^{-1},
\end{aligned}
$$

where $\omega_{L}$ is the angular rate of the gimbal, $K_{t}$ is the torque sensitivity, $K_{e}$ is the back electromotive force (EMF) constant, $R_{m}$ is the motor resistance, $L_{m}$ is the motor inductance, $U$ is the total voltage applied on the motor armature, $T_{M}$ is the torque generated by motor, $T_{d L}$ is the torque disturbances imposed on the payload, including imbalance, cable flexure, friction, and the force between the stator and the rotor of the maglev bearings, $T_{d m}$ is the torque disturbances imposed on the motor, which are caused by bearing friction, cogging, and imperfections in the motor, $N$ is the gear ratio, and $\omega_{b}$ is the angular rate of the base plate [2].

\section{The RBFNN-Based Adaptive Controller Design}

3.1. The State Space Modeling for Single ISP Gimbal. In actual system, the armature inductance $L_{m}$ is so small that it usually can be ignored in the motor model. The angular rate is measured by gyro, while the attitude angular is measured by POS. In order to eliminate the steady error and to compensate for the attitude error caused by gyro drift error, select the state variables as

$$
x=\left[\begin{array}{l}
x_{1} \\
x_{2} \\
x_{3} \\
x_{4}
\end{array}\right],
$$


where $x_{1}=\omega_{L}, x_{2}=\theta_{L}, \dot{x}_{3}=x_{2}$, and $\dot{x}_{4}=x_{3}$. State space equations of single gimbal are as follows:

$$
\begin{aligned}
\dot{x}= & {\left[\begin{array}{c}
\dot{x}_{1} \\
\dot{x}_{2} \\
\dot{x}_{3} \\
\dot{x}_{4}
\end{array}\right] } \\
= & {\left[\begin{array}{ccc}
\frac{-N^{2} K_{t} K_{e}}{J R_{m}} x_{1}+\frac{N K_{t}}{J R_{m}} U+\frac{N^{2} K_{t} K_{e}}{J R_{m}} \omega_{b}+\frac{1}{J} T_{d} \\
x_{1} \\
x_{2} \\
x_{3}
\end{array}\right.} \\
= & \left(\begin{array}{cccc}
\frac{-N^{2} K_{t} K_{e}}{J R_{m}} & 0 & 0 & 0 \\
1 & 0 & 0 & 0 \\
0 & 1 & 0 & 0 \\
0 & 0 & 1 & 0
\end{array}\right)\left[\begin{array}{l}
x_{1} \\
x_{2} \\
x_{3} \\
x_{4}
\end{array}\right] \\
& +\left(\begin{array}{cccc}
\frac{N K_{t}}{J R_{m}} \\
0 \\
0 \\
0
\end{array}\right) U+\left(\begin{array}{ccc}
\frac{N^{2} K_{t} K_{e}}{J R_{m}} & \frac{1}{J} \\
0 & 0 \\
0 & 0 \\
0 & 0
\end{array}\right)\left[\begin{array}{l}
\omega_{b} \\
T_{d}
\end{array}\right] \\
\triangleq & A x+B u+d,
\end{aligned}
$$$$
y=\theta_{L}=x_{2}=\left(\begin{array}{llll}
0 & 1 & 0 & 0
\end{array}\right)\left[\begin{array}{l}
x_{1} \\
x_{2} \\
x_{3} \\
x_{4}
\end{array}\right] \triangleq C x,
$$

where

$$
\begin{aligned}
& A=\left(\begin{array}{cccc}
\frac{-N^{2} K_{t} K_{e}}{J R_{m}} & 0 & 0 & 0 \\
1 & 0 & 0 & 0 \\
0 & 1 & 0 & 0 \\
0 & 0 & 1 & 0
\end{array}\right) \\
& B=\left(\begin{array}{c}
\frac{N K_{t}}{J R_{m}} \\
0 \\
0 \\
0
\end{array}\right) \text {, } \\
& C=\left(\begin{array}{llll}
0 & 1 & 0 & 0
\end{array}\right), \\
& d=\left(\begin{array}{cc}
\frac{N^{2} K_{t} K_{e}}{J R_{m}} & \frac{1}{J} \\
0 & 0 \\
0 & 0 \\
0 & 0
\end{array}\right)\left[\begin{array}{c}
\omega_{b} \\
T_{d}
\end{array}\right], \\
& T_{d}=N T_{d m}+T_{d L}+N(N-1) J_{m} \dot{\omega}_{b}, \\
& J=N^{2} J_{m}+J_{L} .
\end{aligned}
$$

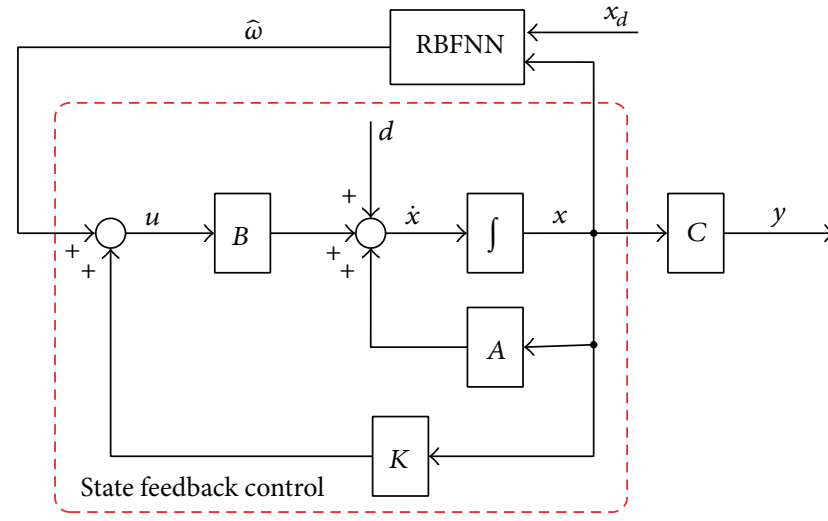

FIGURE 2: The RBFNN-based disturbance compensation scheme.

From model (1), the control input $U$ is used to ensure LOS angular motion accuracy of the gimbal and to compensate the disturbance caused by $\omega_{b}, \dot{\omega}_{b}, T_{d m}$, and $T_{d L}$ at the same time. In order to enhance the accuracy of the MSISP, a RBFNNbased adaptive control approach is proposed. With the classic state feedback control, a disturbance compensator based on RBFNN is designed, which produces an additional equivalent control input based on the current system state and the desired state, thus realizing disturbance compensation (Figure 2).

3.2. The RBFNN-Based Adaptive Controller. The description above shows that the MSISP gimbal system is controllable, and then control input can be divided into two parts: one is the state feedback control input $K x(t)$ and the other is the disturbance compensation input $\widehat{\omega}(t)$, that is,

$$
u(t)=K x(t)+\widehat{\omega}(t)
$$

where $\widehat{\omega}(t)$ is disturbance estimation input generated by the adaptive RBFNN and $K$ is weight matrix of state feedback control.

So, the dynamic model of the ISP gimbal system can be transformed as follows:

$$
\dot{x}(t)=(A+B K) x(t)+B \widehat{\omega}(t)+d(t) .
$$

Then the error equation is as follows:

$$
\dot{e}(t)=(A+B K) e(t)+B \widehat{\omega}(t)-f,
$$

where the error between the desired state variable $x_{d}$ and the real state variable $x$ is denoted as $e=x-x_{d}$. The unknown function $f=\dot{x}_{d}-d(t)-(A+B K) x_{d}$ contains $x_{d}$ and $d(t)$. The disturbance $d(t)$ cannot be measured directly. Since RBFNN has universal approximation capabilities, it can estimate the unknown function $f$ successfully. Therefore, $-d(t)$ can be estimated by $\widehat{\omega}(t)$.

With RBFNN, the unknown function $f$ can be estimated:

$$
f=\bar{W}^{T} s(x)+\bar{\delta}(x),
$$

where $\bar{W}^{T}$ is the ideal neural network weight matrix and $\bar{\delta}$ is the offset vector of neural network output layer. 
Therefore, the error dynamic of the ISP gimbal system in (7) can be transformed as follows:

$$
\dot{e}(t)=(A+B K) e(t)+B \widehat{\omega}(t)-\bar{W}^{T} s(x)-\bar{\delta}(x) .
$$

Using $\widehat{W}$ to approximate $\bar{W}$, the error between the actual value and the ideal RBFNN can be expressed as

$$
\widehat{W}^{T} s(x)-\bar{W}^{T} s(x)=\widetilde{W}^{T} s(x) .
$$

Considering the following control law and RBFNN weighting adaptation law:

$$
\begin{aligned}
\dot{\widehat{W}}_{* i}= & -\Gamma_{i} s_{*}(x) e^{T} P_{* i}, \\
\widehat{\omega}_{i}(t)= & \left(B^{T} B\right)^{-1} B_{i *}^{T} \widehat{W}_{* i}^{T} s_{*}(x) \\
& -\alpha\left(B^{T} B\right)^{-1} B_{i *}^{T} \operatorname{sign}\left(e^{T} P_{* i} B_{i *}\right),
\end{aligned}
$$

where $\widehat{W}_{* i}=\left[\begin{array}{llll}\widehat{W}_{1 i} & \widehat{W}_{2 i} & \cdots & \widehat{W}_{l i}\end{array}\right]^{T}, l$ is the number of hidden nodes of RBFNN, $B_{i *}$ denotes the $i$ th row of $B$, and $s_{*}(x)$ denotes a column vector $\left[\begin{array}{lllll}s_{1}(x) & s_{2}(x) & \cdots & s_{l}(x)\end{array}\right]^{T}$. The disturbance can be approximated and eliminated by the adaptive RBFNN and the tracking error $e \rightarrow 0$, as $t \rightarrow \infty$.

Proof. See the appendix.

\section{Simulations and Experiments}

4.1. Matlab Simulations. The effectiveness of the proposed RBFNN-based disturbance compensation scheme is evaluated by simulation results. Relevant parameters are listed in Table 1 .

In order to verify the proposed scheme and compare different system performances, two different control methods are used in simulation.

(I) Conventional State Feedback Control. Based on the pole assignment theory, the matrices $A \in R^{4 \times 4}$ and $B \in R^{4 \times 1}$ and the state feedback matrix $K \in R^{1 \times 4}$ are selected as follows:

$$
\begin{gathered}
A=\left(\begin{array}{cccc}
-1.2059 & 0 & 0 & 0 \\
1 & 0 & 0 & 0 \\
0 & 1 & 0 & 0 \\
0 & 0 & 1 & 0
\end{array}\right), \\
B=\left(\begin{array}{c}
0.4422 \\
0 \\
0 \\
0
\end{array}\right), \\
K=(-45.3092-4549-1813.3-90.6184) .
\end{gathered}
$$

(II) The RBFNN-Based Disturbance Compensation Scheme. Feedback controller parameters in this scheme are the same as the parameters of (I). In order to reduce calculations, only
TABLE 1: Parameters of a single gimbal system.

\begin{tabular}{lcc}
\hline Parameter & Value & Unit \\
\hline Motor torque constant & $K_{t}=0.414$ & $\mathrm{Nm} / \mathrm{Amp}$ \\
Back EMF constant & $K_{e}=0.407$ & $\mathrm{~V} / \mathrm{rad} / \mathrm{s}$ \\
Armature resistance & $R_{m}=2.6$ & $\mathrm{Ohms}$ \\
Motor inertial & $J_{m}=2.765 \times 10^{-4}$ & $\mathrm{Kg} \mathrm{m}^{2}$ \\
Payload inertial & $J_{L}=2.4$ & $\mathrm{Kg} \mathrm{m}^{2}$ \\
Drive ratio & $N=6.7$ & \\
\hline
\end{tabular}

10 hidden nodes of the adaptive RBFNN are selected, with $\alpha=1.9375$.

According to the previous experience and actual flight tests, the disturbances can be separated as the sinusoidal eccentric disturbances and random friction disturbances [14]. (I) Sinusoidal part: the maximum payload of ISP is $100 \mathrm{~kg}$, with maximum eccentric distance $5 \mathrm{~mm}$ and maximum disturbance translational acceleration $\pm 1 \mathrm{~g}$. As a result, the maximum imbalance torque is $5 \mathrm{Nm}$, and its frequency is selected as the maximum value of aircraft photo flight frequency $(0.5 \mathrm{~Hz})$. (II) Random friction part: the magnitude of the friction torque disturbance is considered as $1 / 4$ of the sinusoid part. Then the external disturbing torque can be described as the following expression:

$$
T_{d}=5 \sin (\pi t)+2.5(\operatorname{rand}(1)-0.5)
$$

where rand(1) stands for a random value within 0 and 1 . Besides, set the base plate angular velocity as $\omega_{b}=1.5 \times$ $\pi / 180 \times \sin (0.5 \times 2 \times \pi \times t) \mathrm{rad} / \mathrm{s}$ (amplitude is $1.5^{\circ}$ and frequency is $0.5 \mathrm{~Hz}$ ). Figure 3 shows the variation curve of disturbing torque.

The curves of attitude angles for two different control methods are shown in Figure 4. The attitude stabilization accuracy of MSISP system controlled by the RBFNN-based control scheme is $0.0169^{\circ}$ (rms), which is $40 \%$ of the value generated by the state feedback control. The maximum attitude errors of the two control schemes are, respectively, $0.067^{\circ}$ and $0.0298^{\circ}$. Additionally, the attitude curve with the proposed control scheme is more smoother than that of the state feedback scheme.

4.2. Experimental Setup. In order to validate the RBFNNbased disturbance compensation method in practical engineering applications, the sway table test is conducted. The MSISP is shown in Figure 5. The dimensions of the MSISP are $753 \mathrm{~mm} \times 630 \mathrm{~mm} \times 225 \mathrm{~mm}$, and its weight is $91.5 \mathrm{~kg}$. Figure 5(c) shows the detail configuration of the maglev bearings system. Different from the mechanical bearing suspension ISP, MSISP can achieve the target of noncontact suspension between the pitch gimbal (3) and the azimuth gimbal (6). (1) is the radial bearing stator, and (2) is the radial bearing rotor. They compose the radial magnetic bearings. (4) and (5) compose the upside axial magnetic bearings pair, while (7) and (8) compose the underside pair. (9) and (10, respectively, are the rotor and stator of the azimuth directdriven motor. 


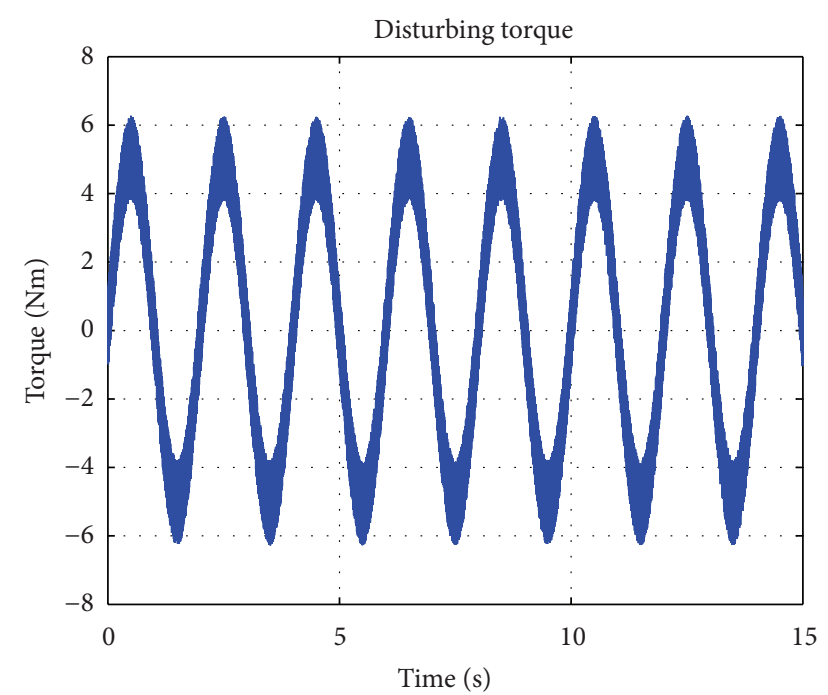

FIGURE 3: The variation curve of disturbing torque.

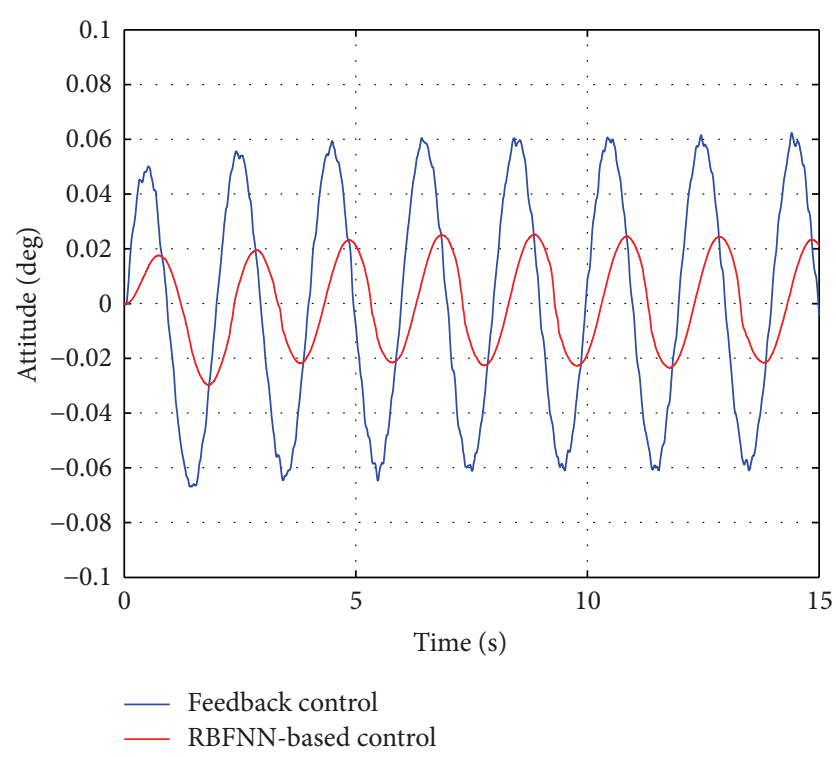

Figure 4: Attitude control curves of the two different control schemes.

Six degrees of freedom sway test table (E-Cue 6241800), produced by Dutch FCS Corporation, can simulate attitude angular motion of the aircraft, and it is capable of handling a gross moving load (GML) of $1800 \mathrm{~kg}$. Therefore, the simulation test environment based on six degrees of freedom is shown in Figure 6. The detail motion parameters are listed in Table 2. The MSISP is mounted on the top of the six degrees of freedom sway table. To validate the control performance, POS AV610, product of Applanix Corporation, is chosen as measurement, whose real-time attitude accuracy is $0.005^{\circ}$ (RMS) [23]. Both ISP gimbal control system and maglev control system adopt floating-point DSP chip TMS320F28335. The servo control frequency reaches to $2 \mathrm{kHz}$. POS AV610 data is stored in the memory card for data processing after experiments. For safety reasons, each system adopts independent power supply. To simulate the airborne sensor, a series of simulated payloads are designed. The simulated payload on the ISP is $90 \mathrm{~kg}$.

4.3. Experimental Results and Analysis. In the experiment, the sway table starts to work with small amplitude and then the amplitude is increased gradually until the expected sway extent. The sway curve of the test table is shown in Figure 7. Both control methods are programmed into ISP control programs, which are distinguished by control command. Different commands bring different control methods and the tested attitude curve for MSISP is shown in Figure 8. From Figures 7 and 8, the isolation role of the ISP can be shown. While the base plate sways largely as shown in Figure 7, the ISP can still hold the attitude of the sensor near $0^{\circ}$ (Figure 8).

Figure 9 shows the comparison curves for two different control methods. Compared with the classic feedback control method, the RBFNN-based control method provides a high performance disturbance observer, so in theory, the performance of the latter scheme is higher than the former. In fact, the classic state feedback control has a certain compensation effect on disturbance, and the proposed RBFNNbased compensation method is used to further compensate the residual disturbance which is beyond the compensation capacity of the state feedback control. Compared with the classic state feedback control, the RBFNN-based compensation method can improve ISP attitude precision significantly. The precision and the maximum attitude angular generated by the RBFNN-based compensation method are $0.0111^{\circ}$ (rms) and $0.046^{\circ}$ that are nearly 43 percent and 53 percent of the values generated by the state feedback control method.

It is proved that the RBFNN-based control method is better than state feedback control on disturbance compensation. A suitable choosing for the node number and parameter $\alpha$ can achieve a much better effect.

\section{Conclusions}

To enhance attitude stabilization accuracy for three-axis MSISP, this paper proposes a novel RBFNN-based control scheme. Compared with the conventional state feedback control, the proposed scheme is effective in engineering application with easy implementation. Both simulations and flight tests validated that the proposed scheme could enhance the attitude stabilization accuracy significantly, and the steady accuracy of the MSISP control system is less than $0.02^{\circ}$ (rms). Moreover, the proposed RBFNN-based control scheme can offer reference for other servo control systems such as turntable control, control moment gyro gimbal control, hard disk drive control, and so on. 
TABle 2: The parameters of the sway test table.

\begin{tabular}{lcccc}
\hline & \multicolumn{2}{c}{ Motion dynamics } & \multirow{2}{*}{ Velocity } \\
\hline Surge & Aypical parameters & $-0.57 \sim+0.57 \mathrm{~m}$ & $\pm 0.7 \mathrm{~m} / \mathrm{s}$ & \multirow{2}{*}{ Acceleration } \\
Sway & $-0.46 \sim+0.57 \mathrm{~m}$ & $\pm 0.50 \mathrm{~m}$ & $\pm 0.7 \mathrm{~m} / \mathrm{s}$ & $\pm 7 \mathrm{~m} / \mathrm{s}^{2}$ \\
Heave & $\pm 0.47 \mathrm{~m}$ & $\pm 0.39 \mathrm{~m}$ & $\pm 0.5 \mathrm{~m} / \mathrm{s}$ & $\pm 7 \mathrm{~m} / \mathrm{s}^{2}$ \\
Roll & $\pm 0.39 \mathrm{~m}$ & $\pm 32.8^{\circ}$ & $\pm 34^{\circ} / \mathrm{s}$ & $>225^{\circ} / \mathrm{s}^{2}$ \\
Pitch & $\pm 32.2^{\circ}$ & $-27.4^{\circ} \sim+31.6^{\circ}$ & $\pm 34^{\circ} / \mathrm{s}$ & $>225^{\circ} / \mathrm{s}^{2}$ \\
Yaw & $-23.2^{\circ} \sim+25.5^{\circ}$ & $\pm 27.6^{\circ}$ & $\pm 35^{\circ} / \mathrm{s}$ & $>225^{\circ} / \mathrm{s}^{2}$ \\
\hline
\end{tabular}

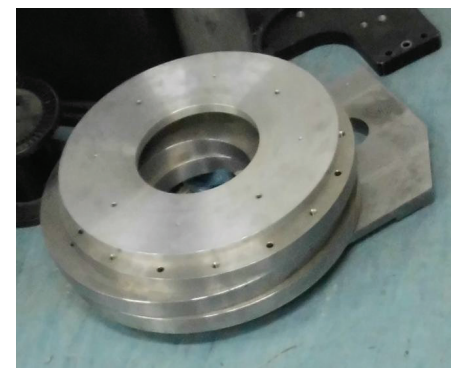

(a) The simulated payload
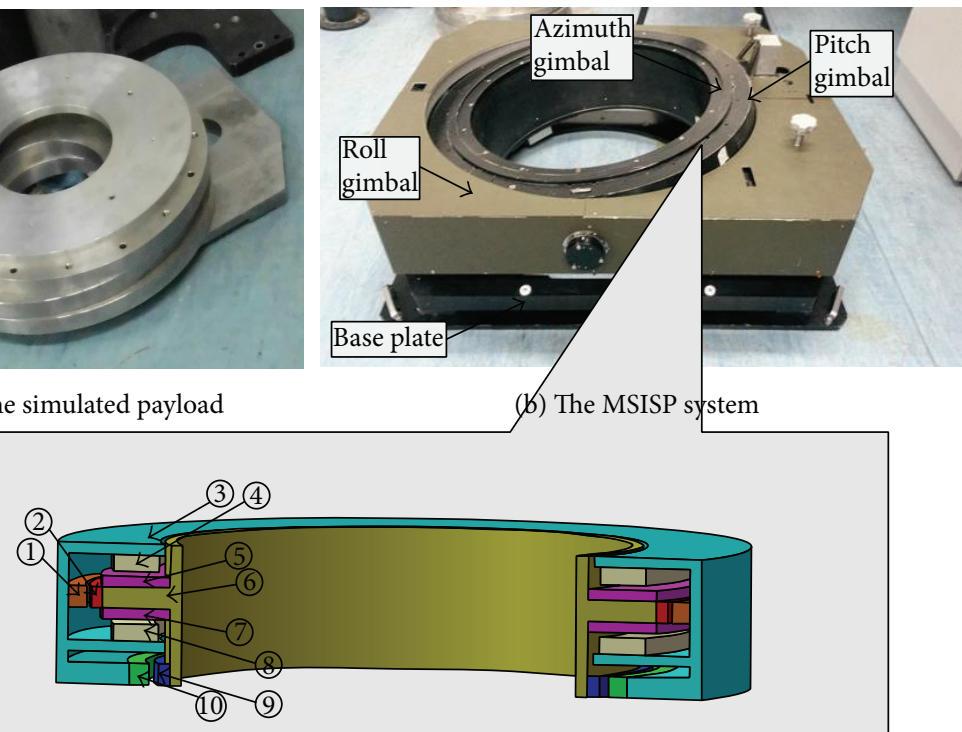

(c) The maglev bearings system configuration

FIGURE 5: The simulated payload and MSISP system.

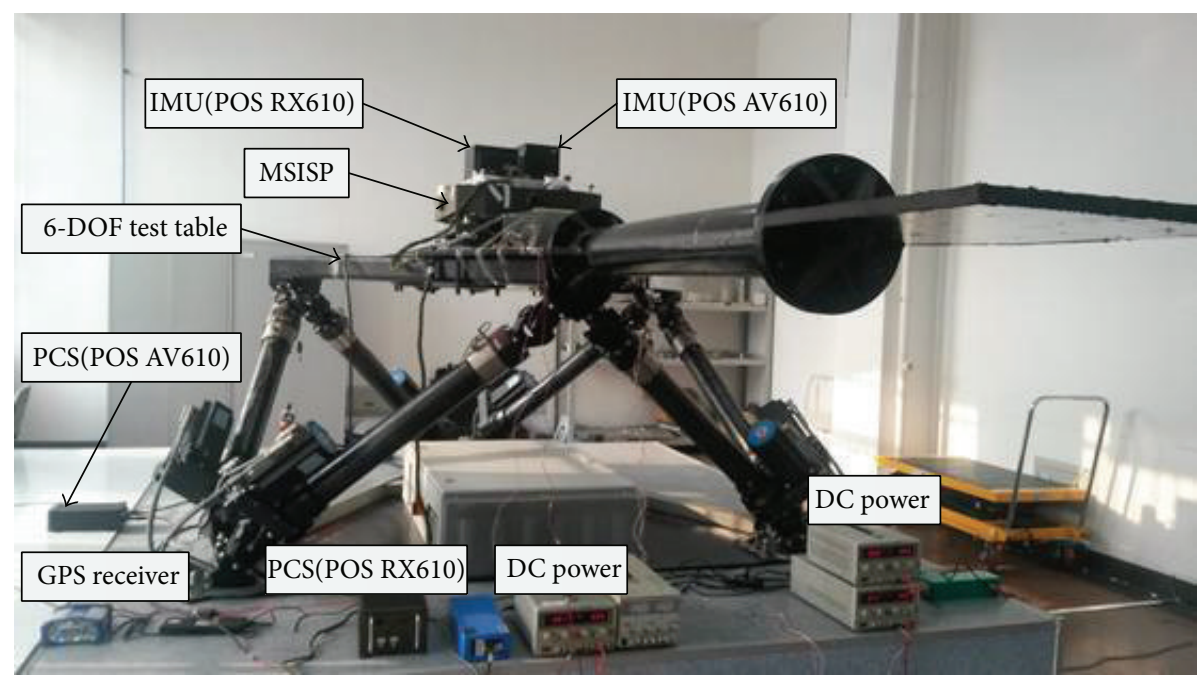

Figure 6: Sway test experiment system. 


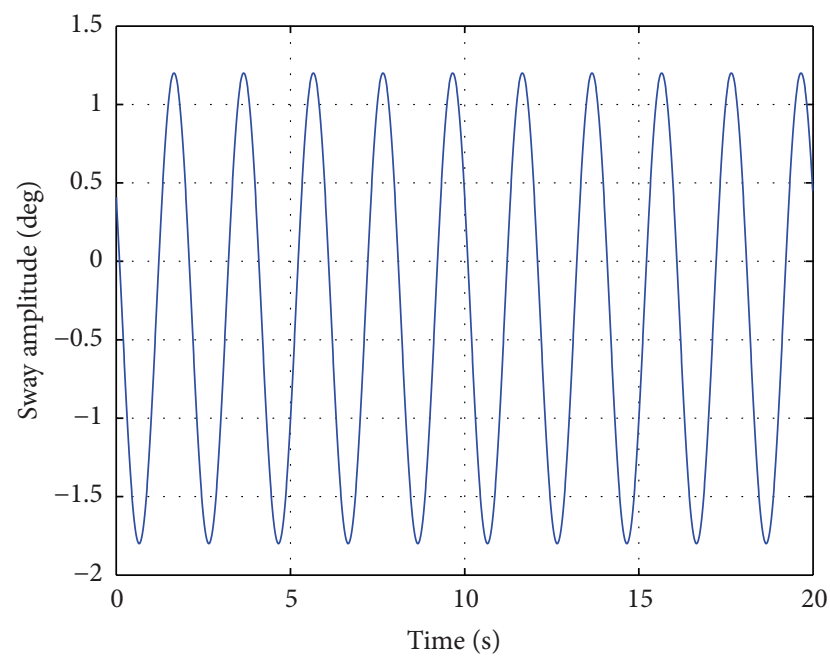

Figure 7: Test table attitude curve.

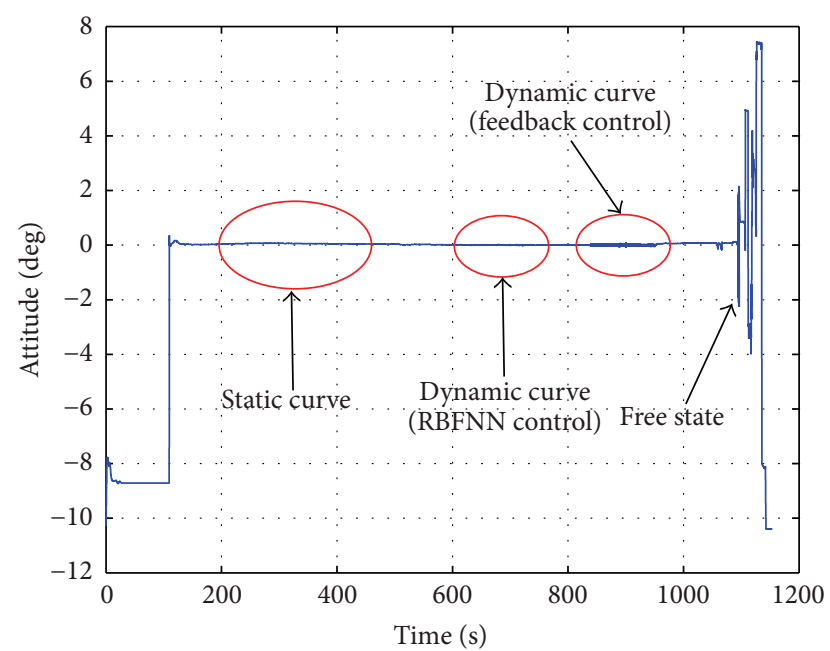

FIGURE 8: Sway experimental attitude wave.

\section{Appendix}

Proof of the asymptotically stable for disturbances estimation and elimination based on adaptive RBFNN.

Proof. Consider the following Lyapunov function:

$$
V=e^{T} P e+\sum_{i=1}^{n} \widetilde{W}_{* i}^{T} \Gamma_{i}^{-1} \widetilde{W}_{* i}
$$

where $\widetilde{W}_{* i}^{T}=\left[\begin{array}{llll}\widetilde{W}_{1 i} & \widetilde{W}_{2 i} & \cdots & \widetilde{W}_{l i}\end{array}\right], l$ is the number of hidden nodes of RBFNN, and $\Gamma_{i}^{-1}$ is a dimensional compatible symmetric positive definite matrix. And the $P$ is the symmetric definite matrix solution to

$$
(A+B K)^{T} P+P(A+B K)=-Q,
$$

where $Q$ is the symmetric definite matrix solution.
Differing the function (A.1),

$$
\begin{aligned}
\dot{V}= & -e^{T} Q e+2 e^{T} P\left(B \widehat{\omega}-\bar{W}^{T} s(x)-\bar{\delta}(x)\right) \\
& +2 \sum_{i=1}^{n} \widetilde{W}_{* i}^{T} \Gamma_{i}^{-1} \dot{\widetilde{W}}_{* i} .
\end{aligned}
$$

Since $\bar{W}$ is a constant value, it is clear that

$$
\dot{\widetilde{W}}=\dot{\widehat{W}} .
$$

Since

$$
\widehat{W}_{* i}^{T} s_{*}(x)-\widetilde{W}_{* i}^{T} s_{*}(x)=\bar{W}_{* i}^{T} s_{*}(x),
$$

therefore, (A.3) is transformed as

$$
\begin{aligned}
& \dot{V}=-e^{T} Q e+2 e^{T} P\left(B \widehat{\omega}-\widehat{W}^{T} s(x)+\widetilde{W}^{T} s(x)-\bar{\delta}(x)\right) \\
& +2 \sum_{i=1}^{n} \widetilde{W}_{* i}^{T} \Gamma_{i}^{-1} \dot{\widehat{W}}_{* i} \\
& =-e^{T} Q e+2\left[\begin{array}{llll}
e^{T} P_{* 1} & e^{T} P_{* 2} & \cdots & e^{T} P_{* n}
\end{array}\right] \\
& \times\left[\begin{array}{c}
B_{1 *} \widehat{\omega}-\widehat{W}_{* 1}^{T} s_{*}(x)+\widetilde{W}_{* 1}^{T} s_{*}(x)-\bar{\delta}_{1}(x) \\
B_{2 *} \widehat{\omega}-\widehat{W}_{* 2}^{T} s_{*}(x)+\widetilde{W}_{* 2}^{T} s_{*}(x)-\bar{\delta}_{2}(x) \\
\vdots \\
B_{n *} \widehat{\omega}-\widehat{W}_{* n}^{T} s_{*}(x)+\widetilde{W}_{* n}^{T} s_{*}(x)-\bar{\delta}_{n}(x)
\end{array}\right] \\
& +2 \sum_{i=1}^{n} \widetilde{W}_{* i}^{T} \Gamma_{i}^{-1} \dot{\widehat{W}}_{* i} \\
& =-e^{T} Q e+2 \sum_{i=1}^{n} e^{T} P_{* i} \\
& \times\left(B_{i *} \widehat{\omega}-\widehat{W}_{* i}^{T} s_{*}(x)+\widetilde{W}_{* i}^{T} s_{*}(x)-\bar{\delta}_{i}(x)\right) \\
& +2 \sum_{i=1}^{n} \widetilde{W}_{* i}^{T} \Gamma_{i}^{-1} \dot{\widehat{W}}_{* i} \\
& =-e^{T} Q e+2 \sum_{i=1}^{n} e^{T} P_{* i}\left(B_{i *} \widehat{\omega}-\widehat{W}_{* i}^{T} s_{*}(x)-\bar{\delta}_{i}(x)\right) \\
& +2 \sum_{i=1}^{n} e^{T} P_{* i} \widetilde{W}_{* i}^{T} s_{*}(x)+2 \sum_{i=1}^{n} \widetilde{W}_{* i}^{T} \Gamma_{i}^{-1} \dot{\widehat{W}}_{* i} \\
& =-e^{T} Q e+2 \sum_{i=1}^{n} e^{T} P_{* i}\left(B_{i *} \widehat{\omega}-\widehat{W}_{* i}^{T} s_{*}(x)-\bar{\delta}_{i}(x)\right) \\
& +2 \sum_{i=1}^{n}\left(\widetilde{W}_{* i}^{T} s_{*}(x) e^{T} P_{* i}+\widetilde{W}_{* i}^{T} \Gamma_{i}^{-1} \dot{\widehat{W}}_{* i}\right) .
\end{aligned}
$$




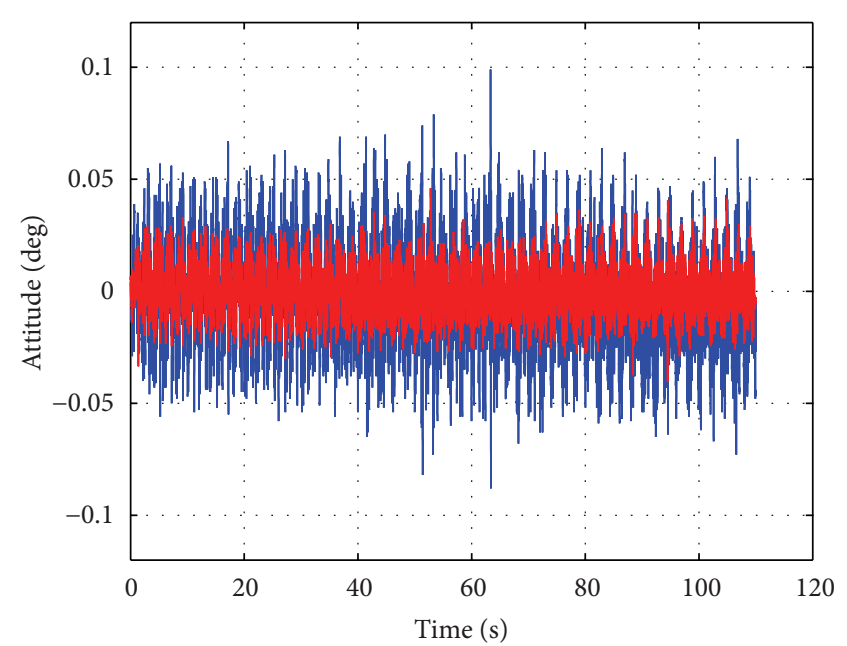

State feedback control RBFNN-based control

(a) Attitude curves of the single ISP gimbal

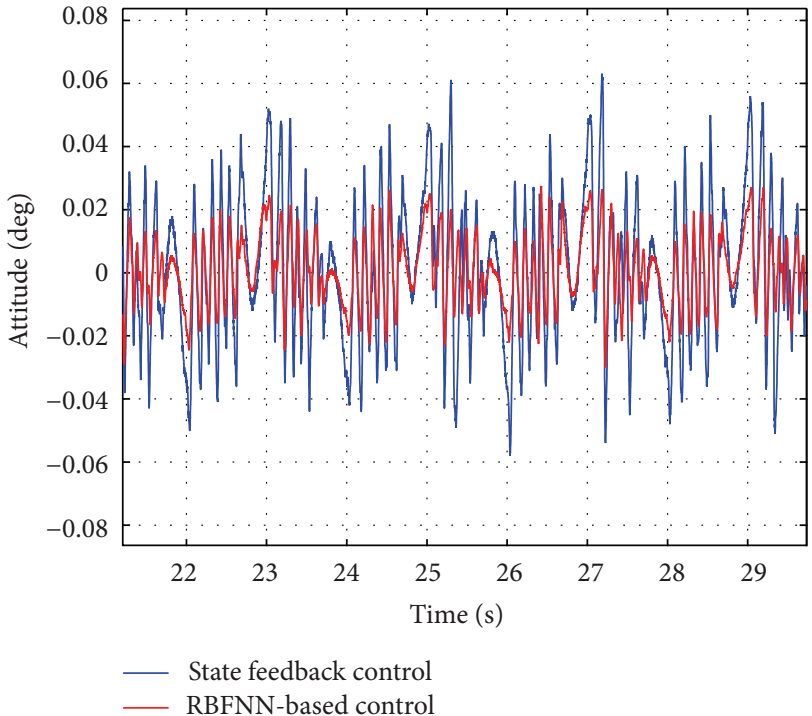

(b) Flight experimental results comparison

FIGURE 9: The comparison of the sway experimental.

Submitting (11) and (12) into the (A.6) and choosing appropriate $\alpha$ to guarantee $\dot{V} \leq 0$

$$
\begin{aligned}
\dot{V} & =-e^{T} Q e+2 \sum_{i=1}^{n} e^{T} P_{* i}\left(B_{i *} \widehat{\omega}-\widehat{W}_{* i}^{T} \mathcal{S}_{*}(x)-\bar{\delta}_{i}(x)\right) \\
& =-e^{T} Q e+2 \sum_{i=1}^{n} e^{T} P_{* i} B_{i *}\left(-\alpha \operatorname{sign}\left(e^{T} P_{* i} B_{i *}\right)-\bar{\delta}_{i}(x)\right) \\
& \leq-e^{T} Q e \leq 0 .
\end{aligned}
$$

The error dynamic model of the ISP gimbal system is asymptotically stable.

Then, it can prove that the tracking error $e \rightarrow 0$, as $t \rightarrow$ $\infty$.

Therefore, with the controller (5), (12), and the RBFNN weighting adaptation law (11), the system (6) is stable. The external disturbance can be approximated and eliminated by the adaptive RBFNN, and the tracking error $e \rightarrow 0$, as $t \rightarrow \infty$ :

$$
\lim _{t \rightarrow \infty}\left[x-x_{d}\right]=0 .
$$

That is, asymptotic tracking is achieved.

\section{Conflict of Interests}

The authors declare that there is no conflict of interests regarding the publication of this paper.

\section{Acknowledgments}

The project is supported in part by the National Basic Research Program of China (973 program; Grant no.
2009CB724001/2), in part by the National Natural Science Foundation of China under Grant 61273033, in part by the Beijing Municipal Natural Science Foundation under Grant 4132043, in part by the National Natural Science Foundation of China under Grant 61374029, and in part by the Beijing Municipal Natural Science Foundation under Grant 61374211.

\section{References}

[1] J. M. Hilkert, "Inertially stabilized platform technology: concepts and principles," IEEE Control Systems Magazine, vol. 28, no. 1, pp. 26-46, 2008.

[2] M. K. Masten, "Inertially stabilized platforms for optical imaging systems: tracking dynamic targets with mobile sensors," IEEE Control Systems Magazine, vol. 28, no. 1, pp. 47-64, 2008.

[3] M. Řezáč and Z. Hurák, "Vibration rejection for inertially stabilized double gimbal platform using acceleration feedforward," in Proceedings of the 20th IEEE International Conference on Control Applications (CCA '11), pp. 363-368, Denver, Colo, USA, September 2011.

[4] Q. Mu, G. Liu, M. Zhong, and Z. Chu, "Imbalance torque compensation for three-axis inertially stabilized platform using acceleration feedforward," in Proceedings of the 8th IEEE International Symposium on Instrumentation and Control Technology (ISICT '12), pp. 157-160, London, UK, July 2012.

[5] Y. Zhang, C. Du, and Q. Mu, "Random error modelling and compensation of accelerometer in airborne remote sensing stabilized platform," Transactions of the Institute of Measurement and Control, vol. 35, no. 4, pp. 503-509, 2013.

[6] http://www.npk-photonica.ru/images/specifications_gsm_3000 .pdf.

[7] http://www.somag-ag.de/index.php?id=23\&L=1.

[8] http://www.leica-geosystems.com/downloads123/zz/airborne/ PAV80/Flyer/Leica_PAV80_Flyer_en.pdf. 
[9] http:// www.leica-geosystems.com/en/Leica-PAV100_103713 .htm.

[10] http://www.geospace.co.za/pdf/DMC\%20Brochure.pdf.

[11] W. Ji, Q. Li, and B. Xu, "Design study of adaptive fuzzy PID controller for LOS stabilized system," in Proceedings of the 9th International Conference on Intelligent Systems Design and Applications (ISDA '06), pp. 336-341, October 2006.

[12] H. J. Hong, P. P. Yun, C. S. Zhao, and Q. Wu, “The application research on fuzzy PI control arithmetic of photoelectric stabilized platform," in Proceedings of the International Workshop on Intelligent Systems and Applications (ISA '09), pp. 1-5, May 2009.

[13] A. R. Amr, S. Chengzhi, F. M. Hany, and W. Tongyu, "Design a robust PI controller for line of sight stabilization system," International Journal of Modern Engineering Research (IJMER), pp. 144-148.

[14] J. M. Hilkert and B. Pautler, "A reduced-order disturbance observer applied to inertially stabilized Line-of-Sight control," in Proceedings of the 25th SPIE Acquisition, Tracking, Pointing, and Laser Systems Technologies, vol. 8052, 2011.

[15] R. D. Mahdy, A. N. Amir, and K. S. Ali, "Predictive output control of a three-axis gyrostabilized platform," Proceedings of the IMechE, Part G: Journal of Aerospace Engineering, pp. 1-11, 2013.

[16] K. C. Tan, T. H. Lee, E. F. Khor, and D. C. Ang, "Design and real-time implementation of a multivariable gyro-mirror lineof-sight stabilization platform," Fuzzy Sets and Systems, vol. 128, no. 1, pp. 81-93, 2002.

[17] J. A. R. Krishna Moorty, R. Marathe, and H. Babu, "Fuzzy controller for line-of-sight stabilization systems," Optical Engineering, vol. 43, no. 6, pp. 1394-1400, 2004.

[18] Y. B. Shtessel, "Decentralized sliding mode control in three-axis inertial platforms," Journal of Guidance, Control, and Dynamics, vol. 18, no. 4, pp. 773-781, 1995.

[19] T. Chen and T. Sheu, "Model reference neural network controller for induction motor speed control," IEEE Transactions on Energy Conversion, vol. 17, no. 2, pp. 157-163, 2002.

[20] M. Mohamadian, E. Nowicki, F. Ashrafzadeh, A. Chu, R. Sachdeva, and E. Evanik, "A novel neural network controller and its efficient DSP implementation for vector-controlled induction motor drives," IEEE Transactions on Industry Applications, vol. 39, no. 6, pp. 1622-1629, 2003.

[21] F. Lin and P. Shen, "Robust fuzzy neural network sliding-mode control for two-axis motion control system," IEEE Transactions on Industrial Electronics, vol. 53, no. 4, pp. 1209-1225, 2006.

[22] J. C. Fang, Z. H. Qi, and M. Y. Zhong, "Feedforward compensation method for three axes inertially stabilized platform imbalance torque," Journal of Chinese Inertial Technology, vol. 18, no. 1, pp. 38-43, 2010.

[23] http://www.optron.com/system-files/applanix-pos-av-dire1304365131.pdf. 


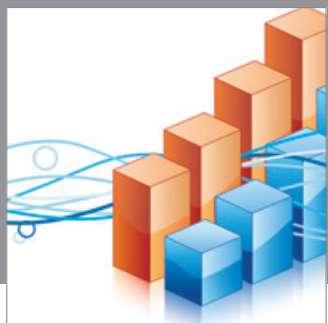

Advances in

Operations Research

mansans

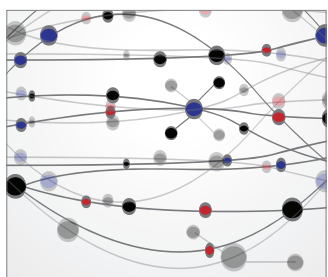

The Scientific World Journal
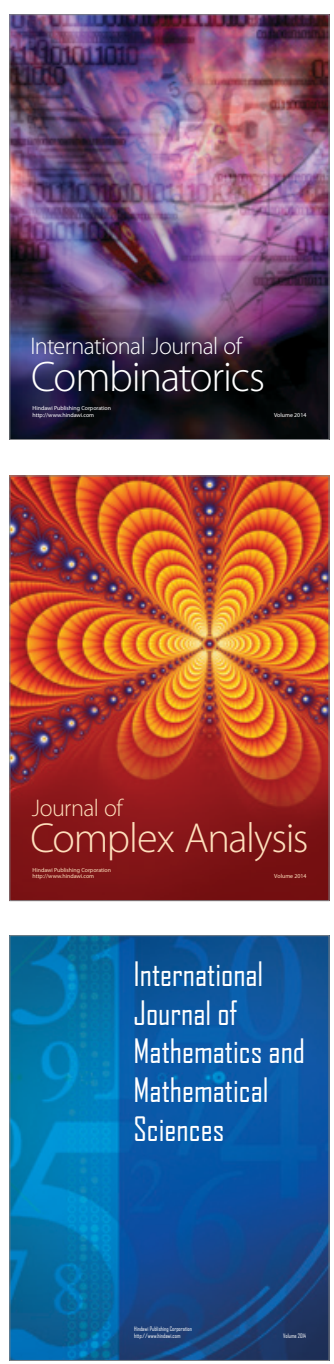
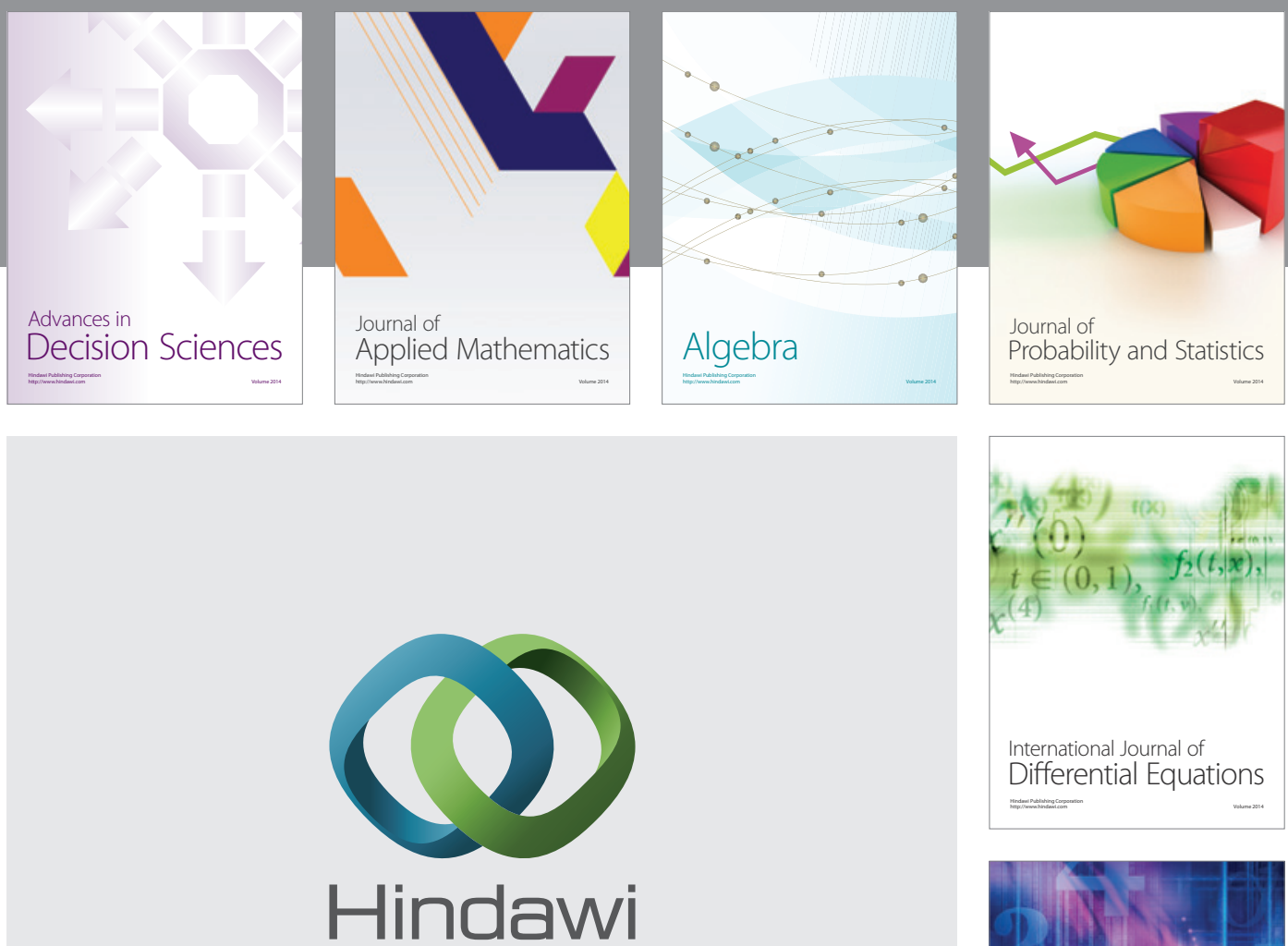

Submit your manuscripts at http://www.hindawi.com
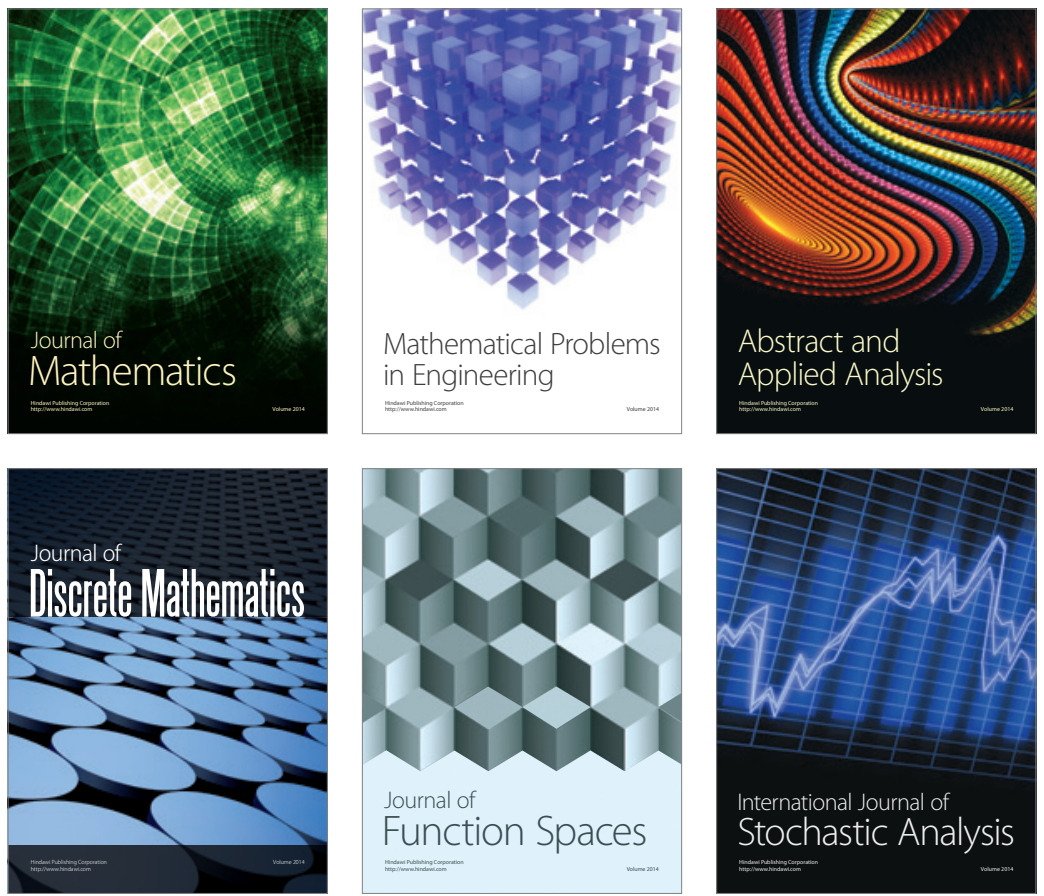

Journal of

Function Spaces

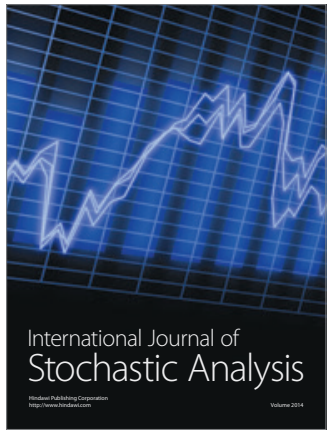

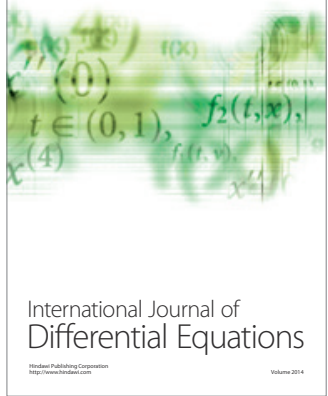
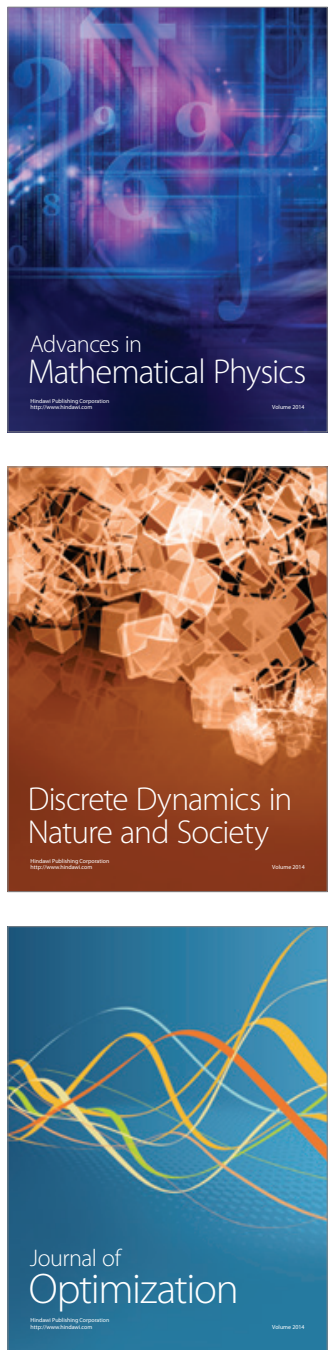